\title{
Effects of shortened host life span on the evolution of parasite life history and virulence in a microbial host-parasite system Thibault Nidelet ${ }^{1,2}$, Jacob C Koella1,3 and Oliver Kaltz ${ }^{* 1,4}$
}

\author{
Address: ${ }^{1}$ UPMC University Paris 06, Laboratoire de Parasitologie Evolutive - UMR 7103, 7 quai St-Bernard, 75252 Paris, France, ${ }^{2}$ UMR de \\ Génétique Végétale, University Paris Sud/INRA/CNRS/INAPG, Ferme du Moulon, 91190 Gif-sur-Yvette, France, ${ }^{3}$ Division of Biology, Imperial \\ College London, Silwood Park Campus, Ascot SL5 7PY, UK and ${ }^{4}$ Institut des Sciences de l'Evolution, UMR 5554, Université de Montpellier 2, CC \\ 065, Place Eugène Bataillon, 34095 Montpellier, France \\ Email: Thibault Nidelet - tnidelet@gmail.com; Jacob C Koella - jkoella@gmail.com; Oliver Kaltz* - oliver.kaltz@univ-montp2.fr \\ * Corresponding author
}

Published: 25 March 2009

BMC Evolutionary Biology 2009, 9:65 doi:I0.1/86/147|-2/48-9-65

This article is available from: http://www.biomedcentral.com/I47I-2/48/9/65

(C) 2009 Nidelet et al; licensee BioMed Central Ltd.

This is an Open Access article distributed under the terms of the Creative Commons Attribution License (http://creativecommons.org/licenses/by/2.0), which permits unrestricted use, distribution, and reproduction in any medium, provided the original work is properly cited.

\begin{abstract}
Background: Ecological factors play an important role in the evolution of parasite exploitation strategies. A common prediction is that, as shorter host life span reduces future opportunities of transmission, parasites compensate with an evolutionary shift towards earlier transmission. They may grow more rapidly within the host, have a shorter latency time and, consequently, be more virulent. Thus, increased extrinsic (i.e., not caused by the parasite) host mortality leads to the evolution of more virulent parasites. To test these predictions, we performed a serial transfer experiment, using the protozoan Paramecium caudatum and its bacterial parasite Holospora undulata. We simulated variation in host life span by killing hosts after I I (early killing) or I4 (late killing) days post inoculation; after killing, parasite transmission stages were collected and used for a new infection cycle.
\end{abstract}

Results: After 13 cycles $(\approx 300$ generations), parasites from the early-killing treatment were less infectious, but had shorter latency time and higher virulence than those from the late-killing treatment. Overall, shorter latency time was associated with higher parasite loads and thus presumably with more rapid within-host replication.

Conclusion: The analysis of the means of the two treatments is thus consistent with theory, and suggests that evolution is constrained by trade-offs between virulence, transmission and withinhost growth. In contrast, we found little evidence for such trade-offs across parasite selection lines within treatments; thus, to some extent, these traits may evolve independently. This study illustrates how environmental variation (experienced by the host) can lead to the evolution of distinct parasite strategies.

\section{Background}

Understanding the factors that shape the evolution of parasite life history and virulence is a major issue in evolutionary biology [1-4], with important implications in applied and medical contexts $[5,6]$. According to standard theory, a parasite needs to exploit the host to increase its rate of transmission. If exploitation harms the host, the parasite must therefore trade current for future transmission and evolution should lead to the balance between virulence and the rate of transmission that maximizes the 
parasite's life-time reproductive success. Where this balance lies may depend on genetic [7], epidemiological [8$11]$ or environmental factors $[12,13]$.

A general prediction concerning environmental factors is that increased extrinsic host mortality (i.e. the mortality that is not due to the parasite) selects for higher virulence [[11,14,16,16], but see [17]]. Because shorter host life span reduces future opportunities of transmission, the constraint to keep the host alive is relaxed. Rather, to compensate for the loss of future transmission, parasites should evolve to grow more rapidly within the host, start transmission earlier and, consequently, be more virulent. This prediction can be restated in terms of classical lifehistory theory: if future reproduction is compromised, we expect selection for earlier age at maturity and increased investment in early reproduction [18]. In this sense, the change in virulence reflects a shift in optimal latency time. A theoretical framework based on this life-history perspective has been developed for the evolution of lysis time in bacteriophages [19]. Such a framework is relevant for medicine, as, from the parasite's point of view, shorter host life span is similar to shortening the infection by the application of a drug treatment. It is therefore important to understand how parasite life-history, such as latency time or fecundity, responds to selection imposed by such a treatment $[20,21]$.

Few experimental studies have investigated the effects of shortened host life span [22-25]. In a serial passage experiment, Cooper et al [22] directly manipulated host life span and timing of transmission of an insect virus by killing infected hosts at two time points after infection. Consistent with theory, early killing selected for higher virulence, possibly caused by more rapid within-host growth. In a similar type of experiment, infecting hamster cells in vitro with vesicular stomatitis virus, Elena [23] found that earlier transmission schedules selected for higher viral population growth rate and increased longevity of viral propagules in the medium, conferring a selective advantage during the early phase of an epidemic. A more complex picture was found in a serial transfer experiment on nematode parasite of rats [25]: with increasing numbers of nematode females inside the host, females from the early killing treatment became less fecund than those from the late killing treatment. Although it was not measured in this study, lower fecundity may also lead to a reduction in virulence. Finally, a clear-cut counterexample to theory was obtained in an experiment on the water flea, Daphina magna, and a microsporidian gut parasite [24]. In the treatment where host death rate was increased, parasites evolved to produce fewer transmission stages and were less virulent than parasites from the control treatment, possibly because the high mortality treatment led to a concomitant increase in the frequency of multiple infections [24]. Thus, these experiments show that parasites indeed respond to selection imposed by a shorter host life-span, but not always by increasing their virulence, as predicted by standard theory. In particular, it remains largely unclear how primary targets of selection, parasite latency time and age at maturity, evolved in these experiments [but see [22]].

We used experimental populations of the bacterial parasite Holospora undulata and its protozoan host, Paramecium caudatum, to investigate effects of early vs. late killing on different aspects of parasite life history. The life cycle of infection [26] is similar to that of certain bacteria-phage systems in that it involves within-host replication with vertical transmission when its host divides and horizontal transmission. However, unlike, e.g., in lytic phages, horizontal transmission does not require host death. Furthermore, this parasite produces two morphologically and functionally distinct forms: reproductive forms for withinhost growth and infectious forms for horizontal transmission. Reproductive forms cannot be transmitted horizontally and infectious forms cannot replicate. The conversion of reproductive into infectious forms seems to rely on a density-dependent switch [27], similar to other parasites, such as Legionella [28] or Trypanosomes [29]. These features give such parasites evolutionary options to respond to shortened host life span that do not necessarily lead to increased virulence. For example, earlier onset of horizontal transmission may be achieved by lowering the threshold density that triggers the production of infectious forms. This can be done without changing withinhost growth rate; moreover, if conversion into infectious forms is irreversible, total parasite load will be lower and thus virulence will be lower (Figure 1).

We experimentally manipulated host life-span in a serial transfer experiment, by killing hosts at two time points. In the early-killing treatment, hosts were killed 11 days after infection and infectious forms of the parasite harvested to start a new infection cycle on previously unexposed hosts. Initially, only a small fraction of the infected hosts produced infectious forms at this time point. In the late-killing treatment, hosts were killed after 14 days, when the majority of infected hosts had begun to produce infectious forms. After 13 infection cycles, we compared parasites from the two treatments for their infectivity, latency time (= time to production of infectious forms), parasite load and virulence. We further examined correlations between these traits among parasite selection lines.

\section{Results}

Adaptation assays were performed on evolved parasite lines from the early- and late-killing treatments. Ancestral parasite lines had been stored at $-80^{\circ} \mathrm{C}$, but their extremely low infection success after thawing precluded 


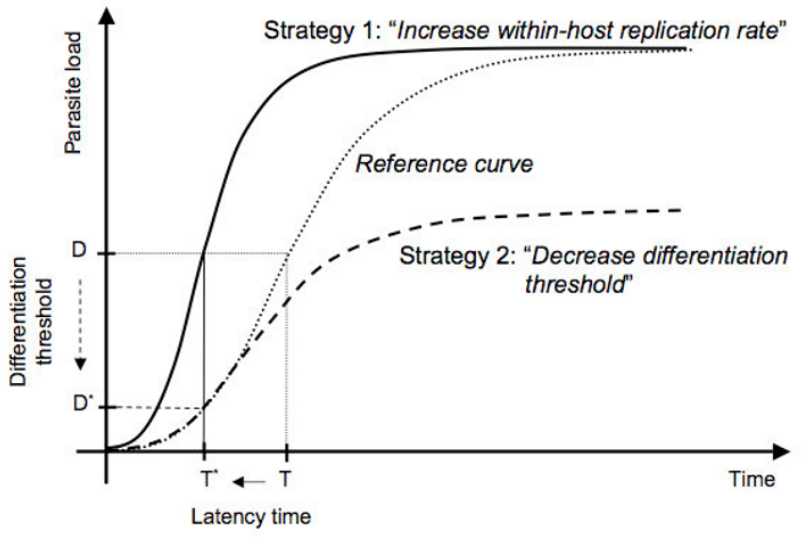

Figure I

Graphical representation of a simple theoretical model illustrating two different possibilities to evolve shorter latency. Latency denotes the time until the onset of conversion of reproductive forms into transmission stages (infectious forms). Suppose a hypothetical ancestral parasite with latency time $T$ (dotted line). Reproductive forms make I division per unit time. At a density-dependent differentiation threshold ( $D=100$ reproductive forms), $60 \%$ of the reproductive forms are converted into infectious forms at each bacterial generation (infectious forms do not multiply). Shorter latency time $\left(T^{*}\right)$ can evolve in two ways. Strategy I: Faster replication of reproductive forms (solid line) reduces the time to the differentiation threshold $D$, thereby reducing latency time $\left(T^{*}\right)$. Consequently, maximum parasite load is also reached earlier. Strategy 2: Replication rate of reproductive forms remains unchanged (dashed line), but the differentiation threshold is reduced $\left(D^{*}\right)$. This shortens latency time $\left(T^{*}\right)$, but also reduces parasite load. If parasite load correlates with consumption of host resources and thus virulence (graphically, the area under the curve), strategy I is associated with higher virulence, whereas strategy 2 is associated with lower virulence. NB: Hosts do not divide in this model; host mortality is nil.

the reconstruction of the parasite founder population for the assay.

\section{Infectivity}

In a dose-controlled inoculation experiment, mean infectivity of parasite selection lines ranged from 18-69\%, except for one outlier from the late-killing treatment (2\%). On average, parasites from the late-killing treatment tended to be more infectious (percentage of infected hosts, without the outlier: $42.1 \pm 5.3 \%$ S.E.) than those from the early-killing treatment $(30.7 \pm 3.7 \%)$, although the difference was not statistically significant $\left(\mathrm{F}_{1,18}=3.05\right.$, $\mathrm{p}=0.0978$; with the outlier: $\mathrm{p}>0.4$ ).

\section{Latency time}

To follow the subsequent development of infection, we sampled infected assay populations at different time points over the course of two weeks. The first hosts producing infectious forms were observed on day 7 after inoculation $(1.6 \%$ of all infected individuals; all other infections were still at the reproductive stage). The proportion of such infectious hosts was significantly higher in populations infected with parasites from the early-killing treatment than in populations infected with parasites from the late-killing treatment (MANOVA on the proportion of infectious forms bearing individuals, day 7-13: $\left.\mathrm{F}_{1,19}=6.38, \mathrm{p}=0.0212\right)$. This effect was most pronounced on day 7 (ANOVA: $\mathrm{F}_{1,18}=5.41, \mathrm{p}=0.0320$ ) and on day 9 $\left(\mathrm{F}_{1,18}=5.48, \mathrm{p}=0.0249\right)$, when there was a $20 \%$ difference in the proportion of infectious hosts (Figure 2). From logistic curve fits, we estimated the time until 50\% of the hosts in a population were producing infectious forms. On average, parasites from the early-killing treatment had reached this point more than half a day earlier (8.81 \pm $0.15 \mathrm{~d})$ than parasites from the late-killing treatment $(9.35$ $\pm 0.19 \mathrm{~d}$; treatment effect: $\mathrm{F}_{1,18}=5.35, \mathrm{p}=0.0328$ ). Thus, early parasites had a shorter latency time.

\section{Parasite within-host growth and production of transmission stages}

First week after infection

Parasite loads increased from $11.5( \pm 0.4)$ reproductive forms on day 2 to $51.1( \pm 1.2)$ on day 7 . Given one division of the host, reproductive forms made 3-4 doublings during this period. One possibility for parasites from the early-killing treatment to shorten their latency time is through faster within-host replication (Figure 1). Indeed,

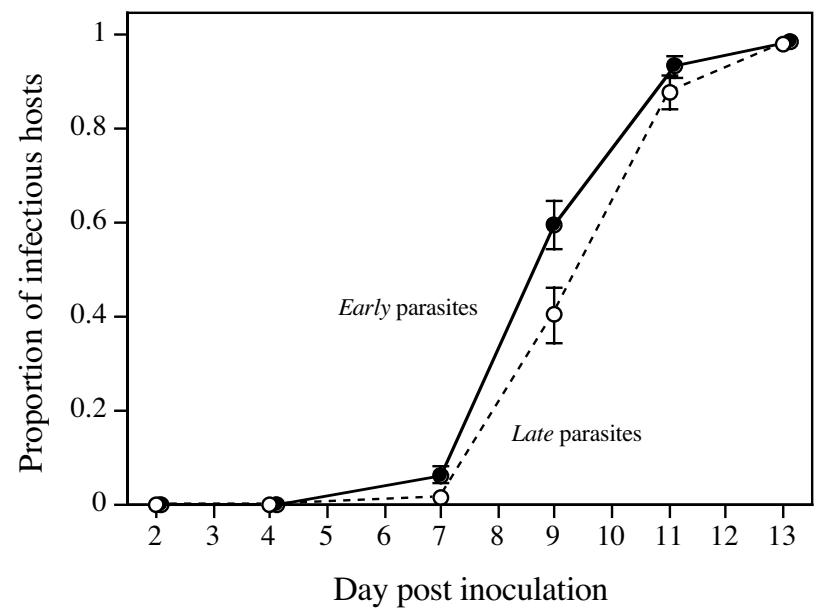

Figure 2

Proportion of infected hosts producing infectious forms over the course of 13 days after inoculation. A higher proportion of infectious hosts indicates a shorter latency time. Values for parasites from early- and late-killing treatments were averaged over selection line means. Error bars represent standard error. 
the mean reproductive parasite load on day 7 was positively correlated with the proportion of individuals producing infectious forms on day 9 (across all assay tubes: $\mathrm{r}$ $=0.40, \mathrm{n}=62, \mathrm{p}=0.0013$ ); thus, the accumulation of reproductive forms in the micronucleus was a good predictor of the onset of the production of infectious forms. This is consistent with the idea of a density-dependent switch from the reproductive to the infectious stage, and thus indicates that faster within-host replication reduces latency time (see also Table 1 ). On day 7 , reproductive loads of parasites from the early-killing treatment were, on average, 5\% larger than those of parasites from the latekilling treatment, but this difference was not significant (MANOVA day 2-7: killing treatment: $\mathrm{F}_{1,19}=0.10, \mathrm{p}=$ 0.7563; treatment $\times$ time interaction: $\mathrm{F}_{2,18}=0.26, \mathrm{p}=$ 0.7764; Figure 3). Thus, there was no statistical support for faster within-host replication of parasites from the early-killing treatment.

\section{Second week after infection}

Shorter latency can also be achieved by decreasing the within-host density threshold. In this case, our model predicts smaller reproductive loads once differentiation into infectious forms has set in (Figure 1). However, when comparing the fraction of hosts still in the exclusively reproductive state, we found no significant difference in reproductive loads between parasites from early- and latekilling treatments (MANOVA days 7-11: $\mathrm{F}_{1,17}=0.20, \mathrm{p}=$ 0.6604 ). If anything, early parasites produced more, rather

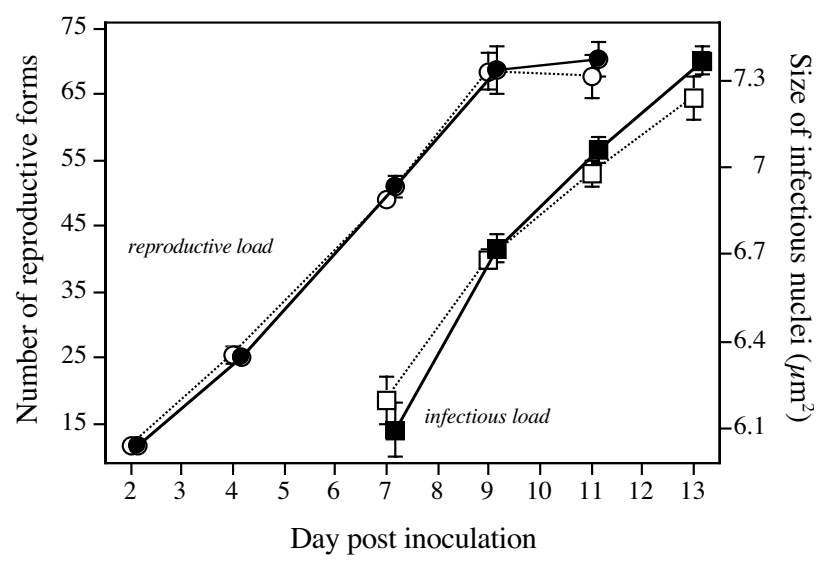

\section{Figure 3}

Reproductive and infectious parasite loads over the course of I 3 days after inoculation. Reproductive loads (left axis) represent the number of reproductive forms per infected individual; infectious loads (right axis) were estimated from the size (length $\times$ width) of nuclei carrying infectious forms. Means for parasites from early- and late-killing treatments were calculated by averaging over individuals within assay tubes, then over assay tubes and selection lines. Error bars represent standard error. than fewer, reproductive forms than did late parasites (Figure 3).

During the second week after infection, infectious forms accumulated in the infected micronculei (Figure 3). Accumulation occurred more rapidly in the hosts infected with parasites from the early-killing treatment, resulting in an approx. $10 \%$ difference in parasite load between the two treatments on day $13\left(\mathrm{~F}_{1,19}=3.20, \mathrm{p}=0.0896\right.$; Figure 3$)$. Overall, earlier onset of the production of infectious forms was associated with larger quantities of infectious forms at the end of the second week $(r=0.41, n=61, p=$ $0.0010)$.

\section{Host density and virulence}

Assay populations (days 2-13 after infection)

Overall, host population density in infected assay tubes was lower than that in the uninfected controls (MANOVA: $\mathrm{F}_{1,64}=5.61, \mathrm{p}=0.0209$ ), with an average reduction of 20$25 \%$ during the last 3 assay dates (day $9,11,13$ ). Population density did not significantly differ between tubes infected with parasites from early- and late-killing treatments $\left(\mathrm{F}_{1,19}=0.61, \mathrm{p}=0.4446\right)$.

\section{Individual experiment (20-40 d after infection)}

In an additional experiment, we measured clonal growth and survival of infected individuals isolated from the assay populations. After 20 days of culture, we obtained a significant treatment effect on final host density $\left(\mathrm{F}_{2,19}=\right.$ $4.29, \mathrm{p}=0.0290$; Figure 4 ). Like in the assay populations, densities were higher in uninfected than infected lines. Moreover, lines infected with parasites from the early-killing treatment had significantly lower densities than those infected with parasites from the early-killing treatment (contrast 'early vs. late': $\mathrm{F}_{1,19}=5.65, \mathrm{p}=0.0285$; Figure 4). This effect was mainly due to a strong difference in survival: the mortality of entire lines was $32.0 \pm 3.8 \%$ for early parasites, but only $17.8 \pm 4.4 \%$ for late parasites $\left(\mathrm{F}_{1,19}=\right.$ 9.21, $\mathrm{p}=0.0068$ ).

\section{Correlations}

Correlations between five characters were analyzed (Table 1 ). When pooling the means of selection lines from the two treatments, we obtained statistically significant overall correlations between latency time and reproductive or infectious parasite loads, with relatively large effect sizes (note that we did not correct p-values for multiple testing [30]). Selection lines with a higher reproductive load (indicating higher within-host replication during the first week) had a shorter latency time (Figure 5a) and larger infectious parasite loads. Shorter latency was also associated with higher final loads. In contrast with the positive association between treatments means, reproductive load was negatively correlated with virulence: selection lines 
Table I: Pearson correlation coefficients for five parasite traits.

\begin{tabular}{|c|c|c|c|c|c|}
\hline Trait & & Reproductive load & Latency time & Infectious load & Virulence \\
\hline \multirow[t]{3}{*}{ Infectivity } & Early & +0.38 & $-0.56(*)$ & $+0.21^{(a)}$ & -0.40 \\
\hline & Late & -0.12 & +0.12 & $+0.45^{(a)}$ & +0.34 \\
\hline & Overall & +0.02 & +0.08 & +0.29 & -0.04 \\
\hline \multirow[t]{3}{*}{ Reproductive load (day 7) } & Early & & $-0.30^{a}$ & $+0.44(a)$ & $-0.75^{* a}$ \\
\hline & Late & & $-0.80 * * a$ & $+0.45(a)$ & $-0.34^{a}$ \\
\hline & Overall & & $-0.55^{*}$ & $+0.47^{*}$ & $-0.38(*)$ \\
\hline \multirow[t]{3}{*}{ Latency time } & Early & & & $-0.6 \mathrm{I}(*)^{\mathrm{a}}$ & $-0.07^{(a)}$ \\
\hline & Late & & & $-0.55(*)^{\mathrm{a}}$ & $+0.5 \mathrm{I}^{(\mathrm{a})}$ \\
\hline & Overall & & & $-0.58 * *$ & +0.01 \\
\hline \multirow[t]{3}{*}{ Infectious load (day I3) } & Early & & & & -0.15 \\
\hline & Late & & & & +0.09 \\
\hline & Overall & & & & +0.14 \\
\hline
\end{tabular}

Correlations were based on the means per parasite selection line and calculated across selection lines within treatments (early-killing, late-killing) and for all selection lines pooled (overall). Within-treatment tests were complemented by Analyses of Covariance (ANCOVA), with treatment as a cofactor, one parasite trait as covariate (line) and another (column) as response variable. For definition of traits, see Methods. Significant correlations marked in bold (uncorrected for multiple testing). Note: ANCOVA can reveal significant relationships, while correlation tests within treatments are non-significant.

$(*) p<0.10 ; * p<0.05 ; * * p<0.01$; main effect of covariate in ANCOVA: (a) $p<0.09$; ${ }^{a} p<0.05$

with a faster accumulation of reproductive loads during the first week after infection tended to be less virulent later on (Figure 5b). These correlations also held across selection lines within treatments (Table 1). The remaining overall correlations had small effect sizes $(|\mathrm{r}|<0.3)$, and in several cases, the within-treatment correlations differed in sign and magnitude (illustrated for latency time and virulence in Figure 5c).

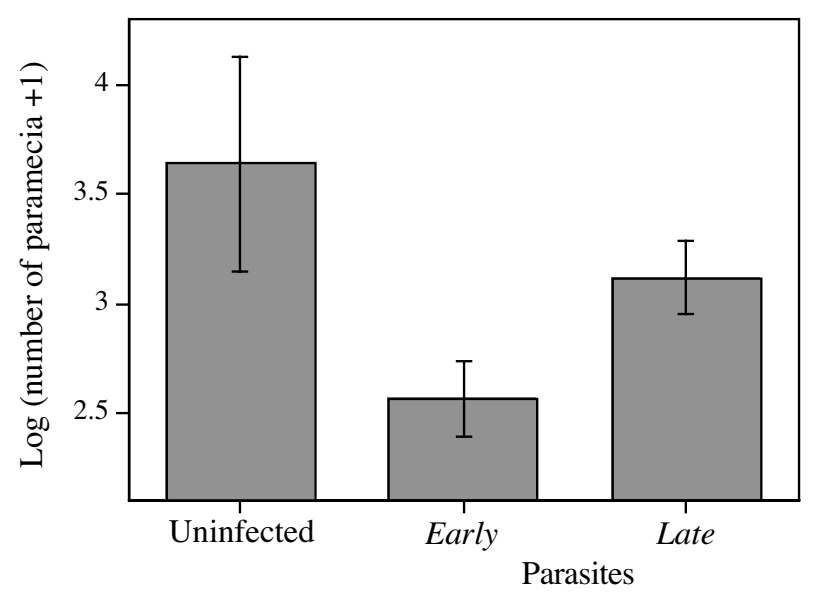

Figure 4

Final host density in the individual assay. Densities measured 20 days after isolation of single individuals. Means for parasites from early- and late-killing treatments were based on selection line means; means for uninfected controls were taken across all assay tubes. Error bars represent standard error.

\section{Discussion}

Life-history theory predicts that cutting off future reproduction selects for increased investment in early reproduction [18]. Consistent with this prediction, parasites from the early-killing treatment (where hosts were killed 11 days after infection) had a shorter latency and accumulated more transmission stages in the micronuclei of infected hosts than did parasites from the late-killing treatment (where hosts were killed 14 days after infection). Early parasites were also more virulent, in agreement with theoretical models predicting that higher virulence evolves under increased background mortality $[11,15]$.

\section{What causes shorter latency time?}

We had hypothesized that parasites, such as $H$. undulata, may reduce latency time by lowering the threshold within-host density that triggers the conversion of reproductive into infectious forms. However, we found no evidence for a lower conversion threshold. In contrast with the predictions under this hypothesis (Figure 1), parasites from the early-killing treatment did not have lower parasite loads, nor were they less virulent than late parasites.

Alternatively, parasites could reduce their latency time by increasing the rate at which they replicate. If the conversion threshold does not change, the more rapid replication would imply earlier conversion (Figure 1). The negative relationship between the densities of reproductive forms after the first week and latency time indicates such a link in our experiment, but there was no clear-cut evidence for faster within-host replication of early parasites. First, the mean density of reproductive forms of early parasites was only marginally larger than that of late par- 

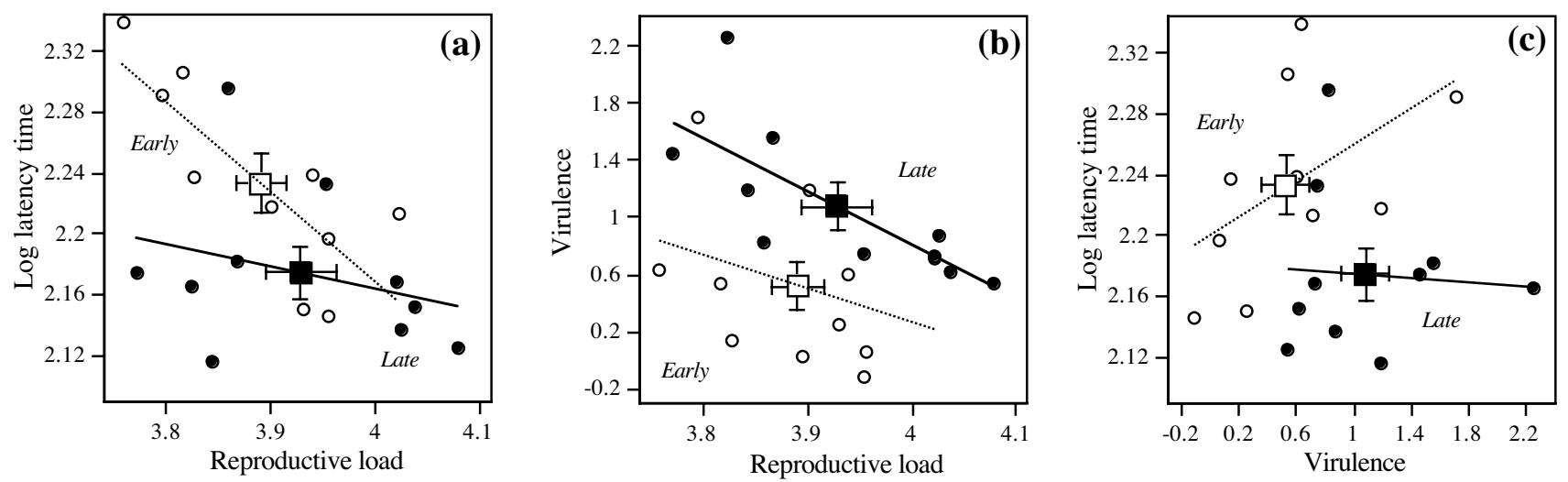

\section{Figure 5}

Within- and between-treatment relationships between mean reproductive parasite loads, latency time and virulence. Each circle represents the mean for a single parasite selection line, averaged over three replicate assay tubes. Black circles denote selection lines from early-killing, open circles selection lines from late-killing treatments. Regression lines illustrate correlations within treatments. Squares indicate the treatment means, averaged over selection line means. Error bars represent standard error. Parasite load = number of reproductive forms per infected individual on day 7 ; latency time $=$ time until $50 \%$ of infected hosts produce infectious forms; virulence $=$ Log-transformed host density uninfected minus log-transformed host density infected in the individual assay (i.e., larger positive values indicate higher virulence).

asites after the first week, and this difference was not statistically significant. Second, at later stages of infection, our measure of parasite load (i.e., the size of the infected nucleus) does not distinguish between reproductive and infectious forms. Therefore, the higher loads of early parasites during the second week may simply reflect their shorter latency time and the concomitant earlier accumulation of infectious forms, irrespective of the rates of replication of reproductive forms. A more conclusive analysis of the within-nucleus dynamics will require a finer temporal resolution of measurements of reproductive loads during the first week after infection and improved techniques allowing us to quantify reproductive forms at later stages of infection.

\section{Virulence: population versus individual assays}

Parasites from the early-killing treatment were more virulent in the assay of individual Paramecium, whereas there was no significant treatment effect in the population assay. Several explanations are possible for this discrepancy. First, host density is strongly reduced only when infectious forms massively accumulate in the infected host [31]. At the time of our final population measurements, although infection was beginning to reduce host population density, parasite loads were moderate. Thus, it was perhaps too early to see a treatment effect, in particular as only a fraction of the populations was infected. Indeed, the test of individuals was performed later on, when infections could even be recognized under the dissecting microscope. Second, in our selection protocol, we killed the entire host population to prepare the inoculum for a new infection cycle. Although this protocol selected for more rapid production of infectious forms, it may have relaxed the pressure for the parasite to kill its host to be transmitted horizontally. As selection for virulence is only relaxed at the time of harvest, an effect of treatment could still be observed at later stages of infection, in the assay of individuals, and thus represent a delayed cost of virulence [32].

\section{Correlations between parasite life-history traits}

Life-history theory relies on evolutionary trade-offs between traits that influence fitness [18]. In host-parasite interactions, the most prominent trade-off is between transmissibility and virulence $[3,33,34]$. In our experiment, the relationships between the means of the earlyand late-killing treatments suggested trade-offs between two components of transmissibility (latency time and infectivity) and virulence. We may interpret our results as correlated sets of evolutionary responses to the different selection pressures imposed by the experimental treatments, leading to separate peaks in an adaptive landscape, possibly determined by a limited number of genes of large effect, as frequently observed in studies on experimental evolution [35].

In part, however, this picture contrasts with the patterns of quasi-genetic correlations observed between the means of individual selection lines (rather than between the two treatment means). Thus, if the means of the selection lines rather than the means of the two treatments were analyzed, there was no evidence a for negative correlation 
between latency time and virulence among selection lines (Figure 5c), reproductive load (and thus presumably within-host replication rate) was negatively correlated with virulence among selection lines within treatments (Figure 5b) (despite a positive correlation between treatment means), and infectious load showed no clear relationship with virulence. These patterns are the opposite of the assumptions of basic theories [34] and differ from results of other studies investigating the experimental evolution of these relationships $[24,36,37]$.

To some extent, the scatter around the treatment means may be non-genetic; we offer three pieces of support for this view. First, we measured parasite loads and virulence in different individuals and at different time points, possibly introducing experimental noise that blurred the underlying genetic correlations. Second, our parasite is not an obligate killer, so that there may be a less straightforward (genetic) link between latency time and virulence than there is between lysis time and virulence in obligately killing bacteriophages [14]. Third, as mentioned above, our experimental protocol may have uncoupled selection pressures on virulence and on latency, allowing the traits to evolve independently and genetically unlinked, at least within the boundaries of the local area around the adaptive peaks. However, the scatter may also represent residual genetic variation, possibly due to genes of minor effect. This variation may be transient. Our results would thus show a snapshot of these lines on their evolutionary pathway towards the adaptive peaks. Alternatively, our snapshot shows individual selection lines moving away from, rather than towards, the adaptive peak. This may reflect additional processes of selection, generating new relationships, such as the negative withintreatment correlations between reproductive load and virulence (Figure $5 \mathrm{~b}$ ). In this case, parallel to the large-scale divergence of treatment means of parasite load and virulence towards different adaptive peaks, local-scale selection may be operating to compensate costs of virulence.

Without more detailed genetic analysis of the evolved lines and the localization of the ancestral parasite population in the adaptive landscape, these interpretations remain speculative. Nonetheless, our results illustrate that the sign of relationships within and among groups or treatments can be different and that interpretations based solely on treatment means can be incomplete or even misleading.

\section{Realism}

The point of our study was to test an evolutionary idea, namely the role of background host mortality in parasite evolution, using a relatively controlled experimental setup. We acknowledge that scenarios of selection in natural populations may be more complex than those in this or related experiments. For example, depending on the source(s) of mortality, selection may not exclusively act on the parasites that are culled from the population [22], nor exclusively on those that remain in the population after culling [24]. Moreover, the selective advantage of shorter-latency genotypes critically depends on epidemiological factors, namely the density of available hosts [38]. So far, however, experimental studies on extrinsic host mortality have not allowed entirely free action of epidemiological dynamics: In our case, we did not interfere with these dynamics prior to the mortality event, but after the event, parasites were provided with new, naïve hosts ad libitum [see also [22,23]]; Ebert and Mangin [24] simply replaced infected hosts with uninfected hosts. Relaxing these experimental constraints may substantially alter evolutionary outcomes. If the parasites from culled hosts remain in the population (e.g., by adding parasites from dead hosts back into the population), the force of infection and levels of coinfection will change. Furthermore, if dead hosts are not artificially replaced, mortality may be compensated by increased birth rates. In our system, for example, increased mortality would be coupled with increased levels of vertical transmission during population re-growth. Thus, selection on the efficacy of vertical and horizontal transmission, as well as evolutionary responses in the host, may act simultaneously (Magalon, Nidelet and Kaltz, unpubl. data). In other words, under more realistic scenarios, it may be difficult to respect the all-else-being-equal rule, and manipulating one factor in question (host mortality) may inevitably produce changes the epidemiology and population dynamics that are not necessarily anticipated by the standard models [24].

\section{Conclusion}

Our study demonstrated an adaptive shift in parasite age at maturity (i.e., latency time), consistent with basic lifehistory theory. As predicted by standard models of virulence evolution, experimentally shortened host life span lead to an evolutionary increase in virulence, confirming results from studies on viral pathogens $[22,23]$. Faster within-host replication is the most likely explanation for the shorter latency time, although this needs to be confirmed by more detailed analysis. However, conclusions about the evolution of genetic correlations or trade-offs between parasite traits should be taken with caution. More generally, our results illustrate how variation in environmental conditions affecting host life-history can feed back on the evolution of parasite life-history. The remaining challenges are experiments testing more realistic scenarios, relaxing constraints on epidemiological and (co)evolutionary processes.

\section{Methods \\ Study organisms}

Paramecium caudatum is a freshwater ciliate [39], with predominant asexual reproduction (mitotic division). The 
diploid micronucleus is similar to a germ line and active mainly during the sexual cycle.

The gram-negative Holospora undulata (alpha-group of the Protobacteria [40]) infects the micronucleus of its host. Infection starts with the uptake of infectious forms (10$15 \mu \mathrm{m})$ from the water. The infectious forms escape from the digestive vacuole and mediate their transfer to the micronucleus. Within $24 \mathrm{~h}$, a single infectious form differentiates into 4 reproductive forms $(5 \mu \mathrm{m})$ that will then start multiplying (initially ca. 1-2 doublings per day). During the first week after infection, only reproductive forms are produced [41]. When bacterial loads further increase, reproductive forms differentiate into infectious forms; possibly, differentiation is triggered by a densitydependent threshold. Infectious forms are released into the environment during mitotic cell division of the host (reproductive forms are transmitted vertically to daughter cells) or upon host death. Accumulating parasite loads produce a heavily swollen micronucleus, filling out the entire individual and packed with several hundreds of infectious forms. At this point, infection strongly reduces cell division and survival of the host [31].

\section{Experimental protocol}

A clonal mass culture [K8, [42]] was infected with a mix of parasites from six selection lines from another long-term experiment [43]. From this mass culture, we created replicate selection lines, each consisting of ca. $10^{4}$ individuals in a $50 \mathrm{ml}$ plastic tube, filled with $35 \mathrm{ml}$ of culture medium ( $1 \mathrm{~g}$ dried organically grown lettuce, ground with mortar and pestle, then autoclaved in $1.5 \mathrm{l}$ of Volvic ${ }^{\mathrm{TM}}$ mineral water [Groupe Danone, France], and inoculated with the bacterium Serratia marcescens [strain A173, Institut Pasteur, Paris, France], as food resource for the paramecia).

Replicate lines were randomly assigned to two treatments. In the early-killing treatment, individuals were killed and infectious forms harvested 11 days after inoculation; in the late-killing treatment, infectious forms were collected 14 days after inoculation. The motivation for our experimental treatments is as follows. First, in the early-killing treatment we intended to impose strong selection on latency time (= onset of production of infectious forms), by killing the population and harvesting the parasite at a time when only few hosts produced infectious forms. In previous experiments [42] as well as in a preliminary test (T. Nidelet, unpubl. data), the frequency of infectious hosts (i.e., carrying at least 1 infectious form) on day 11 ranged from 10 to $30 \%$. Second, in the late-killing treatment, we intended to relax selection on latency time, by killing the population when most infected hosts were already producing large numbers of infectious forms. In most experiments, up to $90 \%$ of the infected hosts were highly infectious on day 14 [e.g., [42]]. We did not choose an even later killing time to avoid potentially confounding selection for parasites with an extremely short latency, which might have allowed them to complete a second infectious cycle at the time of the late-killing harvest.

To harvest infectious forms, $30 \mathrm{ml}$ of a tube $(5 \mathrm{ml}$ were kept as a backup) were centrifuged for $20 \mathrm{~min}$ at $1500 \mathrm{~g}$, $25 \mathrm{ml}$ of the supernatant removed and $1 \mathrm{ml}$ of dimethyl sulfoxide (Sigma, France) was added to kill the paramecia. After two rounds of washing (centrifugation for $20 \mathrm{~min}$ at $1500 \mathrm{~g}$, replacement of supernatant with sterile Volvic), a final volume of $1.5 \mathrm{ml}$ was vortexed for $30 \mathrm{sec}$ in a $2 \mathrm{ml}$ plastic tube, together with several 2-mm glass beads, to grind up the dead paramecia. Thus, the inoculum contained a mix of naturally released and extracted infectious forms.

Approximately $10^{5}$ infectious forms were used to start a new infection cycle, with hosts from the unselected base culture. Paramecia were concentrated in a volume of $5 \mathrm{ml}$ (by centrifugation for $20 \mathrm{~min}$ at $300 \mathrm{~g}$ ) before adding the inoculum. With this protocol, most infections occur during the first $24 \mathrm{~h}$; multiply infected hosts are rare (T. Nidelet, unpubl. data). After $48 \mathrm{~h}$, we added $30 \mathrm{ml}$ of fresh medium, allowing the populations about one doubling until the end of the infection cycle.

Thirteen infection cycles were performed for each of 11 replicate selection lines in each treatment. On three occasions in the early-killing treatment group (cycles 3, 7, 11), the prevalences in certain experimental populations were relatively low. By killing these populations on day 11, we might have harvested an insufficient number of infectious forms to start a new infection cycle. To avoid this problem, we only killed (all) early-killing selection lines on day 14, when infected individuals were producing large amounts of infectious forms. Thus, the early-killing treatment consisted of 13 early-killing rounds and three intermittent late-killing rounds.

\section{Adaptation assay}

During a 14th infection cycle, we compared parasites from early- and late-killing treatments, with three replicate assay tubes per selection line. Three additional assay tubes received a mock inoculum and served as controls. Reconstruction of the parasite founder population from the frozen ancestral lines was not possible because of extremely low infection success of the inocula after thawing.

On day $2,4,7,9,11$ and 13 after inoculation, we measured the density of paramecia by sampling $4 \times 100 \mu \mathrm{l}$ from each tube; ca. 60 individuals were fixed with lacto-acetoorcein [41] to determine the proportion of infected individuals (at $1000 \times$ magnification, phase contrast). For each 
infected individual we recorded the absence or presence of infectious forms in the micronucleus. On days 2 and 4 , we counted the number of reproductive forms in the micronuclei. Later on, we took the dimensions of the infected micronucleus (length $\times$ width in $\mu \mathrm{m}$ ) as an estimate of parasite load. During the first week after infection, this measure gives a good approximation of the number of reproductive forms in the micronucleus; at later stages of infection, it mainly reflects the number of infectious forms [27].

On day 20 after infection, we started an additional experiment to measure parasite virulence. We isolated five infected individuals from each infected assay tube (except for five assay tubes that were omitted due to handling error) as well as five uninfected individuals from each control assay tube. Individuals were placed singly in 200 $\mu \mathrm{l}$ of fresh medium in $500 \mu \mathrm{l}$ plastic tubes, with fresh medium $(100 \mu \mathrm{l})$ added once, on day 10 after isolation. On day 20 after isolation, we determined the density of paramecia in each tube. The density in infected assay tubes relative to uninfected controls is a measure of parasite virulence, integrating effects of infection on host division and survival.

\section{Statistical analysis}

Infections with one parasite selection line from the earlykilling treatment failed to develop for unknown reasons. We had observed normal development of this line in all previous infection cycles and therefore decided to exclude the three assay tubes of this selection line from the analyses. Of the remaining 63 inoculated assay tubes, one was found uninfected and grouped with the three control assay tubes. For these tubes, we analyzed treatment effects on the following traits:

i) Infectivity: the proportion of infected hosts, combined over days 2, 4 and 7 .

ii) Parasite load: in the reproductive state, parasite load was calculated as the mean number of reproductive forms for hosts not producing infectious forms; parasite load in the infectious state was estimated as the mean nucleus size $\left[\mu \mathrm{m}^{2}\right]$ of hosts producing infectious forms.

iii) Latency time: For each assay date, we calculated the proportion of infectious individuals (i.e., that produced infectious forms) in a population; this proportion reflects the timing of the onset of the production of infectious forms and thus latency time. We also used logistic curve fits to estimate the time until $50 \%$ of the infected individuals produced infectious forms. This time was taken as a correlate of latency time.

iv) For effects of infection on host growth and survival (virulence), we compared final host population size in the assay tubes (day 13 after infection) and final densities in the assay of individual paramecia (day 20 after isolation). Virulence in the individual assay was calculated as: $\log ($ mean density +1 , uninfected $)-\log ($ mean density +1 , infected); this difference was calculated for each replicate, then averaged over assay tubes and parasite selection lines. Note that for 4 assay tubes (from 4 different parasite selection lines) in the individual assay, we had established fewer than the required five replicates; to obtain reliable estimates at the level of the assay tube, these replicates ( $\mathrm{n}$ $=10$ ) were excluded from analysis.

Using the JMP statistical package [44], we carried out nested ANOVAs for single assay dates, with killing treatment and selection line(within killing treatment) as explanatory variables. We also report results from repeated-measures MANOVAs, carried out on the averages over the three replicate tubes per parasite selection line. Where necessary, we used arcsine transformation of proportions and log-transformations of parasite load to meet the assumptions of normality and homogeneity of variance. Finally, we examined pairwise correlations between the above traits. Correlations were analyzed across all selection lines pooled, as well as across selection lines within treatments (the latter complemented by Analyses of Covariance).

\section{Authors' contributions}

TN carried out the selection experiment and the adaptation assay and participated in the experimental design of the study, statistical analysis and drafting of the manuscript. JC participated in the experimental design of the study and the drafting of the manuscript. OK participated in the experimental design of the study, the preparation of the selection experiment, statistical analysis and the drafting of the manuscript. All authors read and approved the final manuscript.

\section{Acknowledgements}

We thank Angus Buckling, Mike Hochberg, Hélène Magalon, Elena Arriero and Nicolas Mouquet for discussion and two anonymous reviewers for their comments. This work was financed by a grant "ACl Jeunes Chercheurs" (no. 035167) to O.K

\section{References}

I. Combes C: Parasitism: The Ecology and Evolution of Intimate Interactions. Chicago: University Of Chicago Press; 200 I.

2. Frank SA: Models of parasite virulence. $Q \operatorname{Rev} B i o l$ 1996, $71: 37-77$.

3. Bull J]: The evolution of virulence. Evolution 1994, 48: I 423-I437.

4. Koella JC, Agnew P, Michalakis Y: Coevolutionary interactions between host life histories and parasite life cycles. Parasitology 1998, I 1 6:S47-S55.

5. Dieckmann U, Metz JAJ, Sabelis MW, Sigmund K, (eds): The Adaptive Dynamics of Infectious Diseases: In Pursuit of Virulence Management. Cambridge: Cambridge University Press; 2002.

6. Stearns SC, Koella JC, (eds): Evolution in health and disease. Oxford, UK: Oxford University Press; 2007.

7. Regoes RR, Nowak MA, Bonhoeffer S: Evolution of virulence in a heterogeneous host population. Evolution 2000, 54:64-7I.

8. van Baalen M: Coevolution of recovery ability and virulence. Proc R Soc London, B 1998, 265:317-325. 
9. Lipsitch M, Herre EA, Nowak MA: Host population structure and the evolution of virulence: a "law of diminishing returns". Evolution 1995, 49(4):743-748.

10. Brown SP, Hochberg ME, Grenfell BT: Does multiple infection select for raised virulence? Trends Microbiol 2002, I0(9):40I-405.

II. van Baalen M, Sabelis MW: Dynamics of multiple infection and the evolution of virulence. Am Nat 1995, I 46:88I-910.

12. Hochberg ME, van Baalen M: Antagonistic coevolution over productivity gradients. Am Nat 1998, I 52:620-634.

13. Koella JC, Restif O: Coevolution of parasite virulence and host life histories. Ecol Lett 200I, 4:207-2I4.

14. Ebert D, Weisser WW: Optimal killing for obligate killers: the evolution of life histories and virulence of semelparous parasites. Proc R Soc London, B I997, 264(1 384):985-99I.

15. May RM, Anderson RM: Epidemiology and genetics in the coevolution of parasites and hosts. Proc $R$ Soc London, B 1983, 219:28I-3I3.

16. Choo K, Williams PD, Day T: Host mortality, predation and the evolution of parasite virulence. Ecol Lett 2003, 6(4):310-315.

17. Williams PD, Day T: Interactions between sources of mortality and the evolution of parasite virulence. Proc $R$ Soc London, $B$ 200I, 268( ( 1 483):233I-2337.

18. Stearns SC: The evolution of life histories. Oxford: Oxford University Press; 1992.

19. Bull JJ, Pfennig DW, Wang I-N: Genetic details, optimization and phage life histories. Trends Ecol Evol 2004, 19:76-82.

20. Poulin R: Evolutionary Ecology of Parasites: From Individuals to Communities. London: Chapman \& Hall; 1998.

21. Skorping A, Read AF: Drugs and parasites: global experiments in life history evolution? Ecol Lett 1998, I:I0-12.

22. Cooper VS, Reiskind MH, Miller JA, Shelton KA, Walther BA, Elkinton JS, Ewald PW: Timing of transmission and the evolution of virulence of an insect virus. Proc $R$ Soc London, B 2002, 269(|496): | |6|-||65

23. Elena SF: Evolutionary history conditions the timing of transmission in vesicular stomatitis virus. Inf Gen Evol 200I, I(2): I5I-I59.

24. Ebert $D$, Mangin KL: The influence of host demography on the evolution of virulence of a microsporidian gut parasite. Evolution 1997, 5 I: | 828-1837.

25. Paterson S, Barber R: Experimental evolution of parasite lifehistory traits in Strongyloides ratti (Nematoda). Proc Biol Sci 2007, 274( (1617): 1467-1474.

26. Görtz H-D, Brigge T: Intracellular bacteria in protozoa. Naturwissenschaften 1998, 85:359-368.

27. Kaltz $\mathrm{O}$, Koella JC: Host growth conditions regulate the plasticity of horizontal and vertical transmission in Holospora undulata, a bacterial parasite of the protozoan Paramecium caudatum. Evolution 2003, 57:1489-1497.

28. Molofsky AB, Swanson MS: Differentiate to thrive: lessons from the Legionella pneumophila life cycle. Mol Microbiol 2004 53(I):29-40

29. Matthews KR, Ellis JR, Paterou A: Molecular regulation of the life cycle of African trypanosomes. Trends Parasitol 2004 20(1):40-47.

30. García LV: Escaping the iron claw in ecological studies. Oikos 2004, 105:657-663.

31. Restif O, Kaltz O: Condition-dependent virulence in a horizontally and vertically transmitted bacterial parasite. Oikos 2006 I I 4: | 48 - I 58.

32. Day $\mathrm{T}$ : Virulence evolution and the timing of disease life-history events. Trends Ecol Evol 2003, I8: I I3-1 I8.

33. Ebert $D$, Bull J]: Challenging the trade-off model for the evolution of virulence: is virulence management feasible? Trends Microbiol 2003, II (I): 15-20

34. Lipsitch M, Moxon ER: Virulence and transmissibility: what is the relationship? Trends Microbiol 1997, 5:31-37.

35. Bell G: Selection: the mechanism of evolution. Oxford: Oxford University Press; 2008.

36. Turner PE, Vaughn SC, Lenski RE: Tradeoff between horizontal and vertical modes of transmission in bacterial plasmids. Evolution 1998, 52:315-329.

37. Messenger S, Molineux I, Bull J: Virulence evolution in a virus obeys a trade-off. Proc $R$ Soc London, B 1999, 266:397-404.
38. Abedon ST, Hyman P, Thomas C: Experimental examination of bacteriophage latent-period evolution as a response to bacterial availability. Appl Environ Microbiol 2003, 69(I 2):7499-7506.

39. Wichtermann RT: The Biology of Paramecium. New York: Plenum Press; 1986.

40. Amann R, Springer N, Ludwig W, Görtz H-D, Schlaifer K-H: Identification in situ and phylogeny of uncultured bacterial endosymbionts. Nature 1991, 35 I:161-164.

41. Görtz H-D, Dieckmann J: Life cycle and infectivity of Holosporo elegans Haffkine, a micronucleus-specific symbiont of Paramecium caudatum (Ehrenberg). Protistologia 1980, 16:591-603.

42. Fels $D$, Kaltz O: Temperature-dependent transmission and latency of Holospora undulata, a micronucleus-specific parasite of the ciliate Paramecium caudatum. Proc $R$ Soc London, $B$ 2006, 273: 1031-1038.

43. Lohse K, Gutierrez A, Kaltz O: Experimental evolution of resistance in Paramecium caudatum against the bacterial parasite Holospora undulata. Evolution 2006, 60: I I77-II86.

44. SAS: JMP statistics and graphics guide (version 5.0.1.2). Cary, N.C.: SAS Institute; 2003.

Publish with Biomed Central and every scientist can read your work free of charge

"BioMed Central will be the most significant development for disseminating the results of biomedical research in our lifetime. "

Sir Paul Nurse, Cancer Research UK

Your research papers will be:

- available free of charge to the entire biomedical community

- peer reviewed and published immediately upon acceptance

- cited in PubMed and archived on PubMed Central

- yours - you keep the copyright
BioMedcentral 